IUCrJ

ISSN 2052-2525

CRYO|EM

Received 14 May 2019

Accepted 16 August 2019

Edited by L. A. Passmore, MRC Laboratory of Molecular Biology, UK

Keywords: cryo-EM; electron cryo-microscopy; radiation damage; helium; liquid-helium cooling; beam-induced motion; beam-induced movement; apoferritin.

EMDB references: apoferritin, data collected at liquid-helium temperature, EMD-4698; data collected at liquid-nitrogen temperature, EMD-4701

Supporting information: this article has supporting information at www.iucrj.org
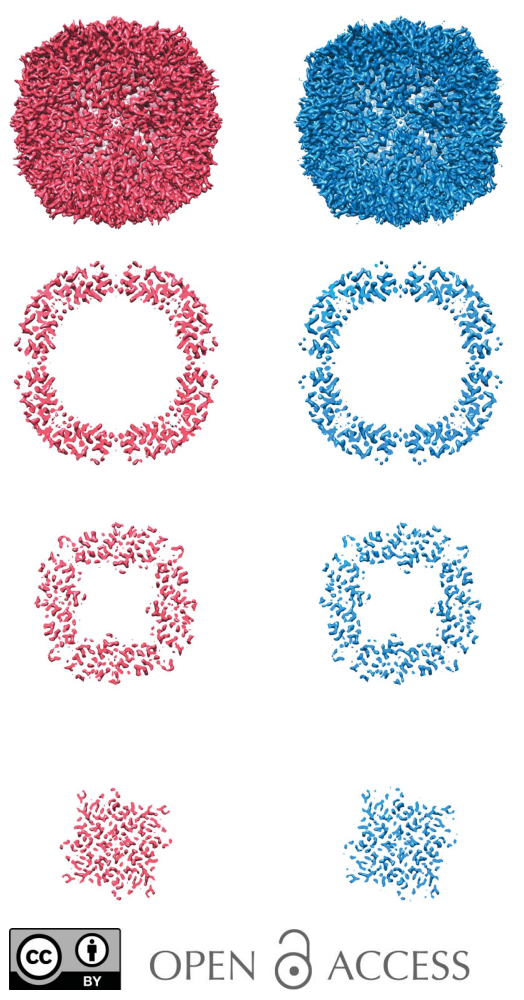

\section{A comparative study of single-particle cryo-EM with liquid-nitrogen and liquid-helium cooling}

\author{
Olivia Pfeil-Gardiner, Deryck J. Mills, Janet Vonck and Werner Kuehlbrandt*
}

Department of Structural Biology, Max Planck Institute of Biophysics, Max-von-Laue-Strasse 3, 60438 Frankfurt, Germany. *Correspondence e-mail: wekuehlb@biophys.mpg.de

Radiation damage is the most fundamental limitation for achieving high resolution in electron cryo-microscopy (cryo-EM) of biological samples. The effects of radiation damage are reduced by liquid-helium cooling, although the use of liquid helium is more challenging than that of liquid nitrogen. To date, the benefits of liquid-nitrogen and liquid-helium cooling for single-particle cryo-EM have not been compared quantitatively. With recent technical and computational advances in cryo-EM image recording and processing, such a comparison now seems timely. This study aims to evaluate the relative merits of liquidhelium cooling in present-day single-particle analysis, taking advantage of direct electron detectors. Two data sets for recombinant mouse heavy-chain apoferritin cooled with liquid-nitrogen or liquid-helium to 85 or $17 \mathrm{~K}$ were collected, processed and compared. No improvement in terms of resolution or Coulomb potential map quality was found for liquid-helium cooling. Interestingly, beaminduced motion was found to be significantly higher with liquid-helium cooling, especially within the most valuable first few frames of an exposure, thus counteracting any potential benefit of better cryoprotection that liquid-helium cooling may offer for single-particle cryo-EM.

\section{Introduction}

Electron microscopy (EM) has become a key technique for determining the structures of biological macromolecules at high resolution (Vinothkumar \& Henderson, 2016; Quentin \& Raunser, 2018; Cheng, 2015; Kühlbrandt, 2014a; Bai et al., 2015; Scapin et al., 2018), an essential step in understanding biological processes at the molecular level. The most fundamental and ultimately insurmountable limitation of the method is radiation damage to the sample. Biological samples tolerate only low electron doses, which limits the signal-tonoise ratio of the electron micrographs recorded. Cooling the sample reduces the effective damage per scattered electron significantly (Stark et al., 1996; Fujiyoshi, 1998; Jeng \& Chiu, 1984; Hayward \& Glaeser, 1979), which is one reason why vitrified biological samples are routinely cooled, most often with liquid nitrogen $\left(\mathrm{IN}_{2}\right.$, boiling temperature $\left.77 \mathrm{~K}\right)$, for electron cryo-microscopy (cryo-EM). Studies of twodimensional (2D) protein crystals have shown that liquidhelium (lHe) cooling (down to $4 \mathrm{~K}$ ) limits radiation damage even further, since high-order diffraction spots of protein crystals fade more slowly at lower temperatures (Stark et al., 1996; Fujiyoshi, 1998). Electron microscopes have been developed specially to allow $1 \mathrm{He}$ cooling (Fujiyoshi et al., 1991) and a number of 2D crystal structures have been determined using them. In 1990, Henderson and coworkers determined the structure of bacteriorhodopsin to a resolution of $3.5 \AA$ using data collected mostly at lHe temperature (Henderson et al., 1990). The $3.4 \AA$ resolution structure of 
light-harvesting complex II was then determined using only data acquired at lHe temperature (Kühlbrandt et al., 1994). In successive years, high-resolution $(\leq 4 \AA)$ structures have been determined of various aquaporins (Murata et al., 2000; Tani et al., 2009; Hiroaki et al., 2006; Gonen et al., 2005), bacteriorhodopsin again (Kimura et al., 1997), nicotinic acetylcholine receptor (Miyazawa et al., 2003; Unwin, 2005) and glutathione transferase (Holm et al., 2006), all using $1 \mathrm{He}$ cooling. For single-particle EM, helium cooling has been used only rarely (Sato et al., 2001; Ludtke et al., 2008; Jiang et al., 2008), mostly because it resulted in an unexpected, and unexplained, loss of contrast (Danev \& Nagayama, 2008; Zhou, 2008). In 2010, Bammes and coworkers reported a study with Fourier-transformed bright-field images of thin catalase crystals cooled with $1 \mathrm{He}$, encountering 'abnormal behaviour' of Bragg peaks at $4 \mathrm{~K}$ (Bammes et al., 2010). The intensities of some peaks were found to increase upon radiation before decreasing as expected. A similar behaviour had previously been observed for catalase crystals at room temperature (Unwin \& Henderson, 1975). Electron cryo-tomography (cryo-ET) has revealed that biological samples cooled with $\mathrm{lHe}$ show extensive beam-induced movement and tend to bubble, suggesting that $\mathrm{lHe}$ cooling is disadvantageous for cryo-ET (Iancu et al., 2006; Comolli \& Downing, 2005). It is now thought that radiolytic fragments, which can diffuse out of the sample at liquid-nitrogen $\left(\mathrm{IN}_{2}\right)$ temperature, are frozen into the sample at lower temperatures and accumulate within it, resulting in an expansion of the sample volume, with concomitant beam-induced movement and reduced image contrast (Iancu et al., 2006; Meents et al., 2010). The effect was apparent at the comparatively high electron doses (a few $100 \mathrm{e}^{-} \AA^{-2}$ ) that are required for electron tomography. The doses applied in single-particle cryo-EM are significantly lower (typically around $50 \mathrm{e}^{-} \AA^{-2}$ ). To date, no systematic comparisons of $1 \mathrm{He}$ and $\mathrm{IN}_{2}$ cooling in single-particle cryo-EM have been published. In view of the recent advances in terms of detector speed and sensitivity (Kühlbrandt, 2014b), specimen-support stability (Russo \& Passmore, 2014b) and correction for beam-induced motion by image processing (Zivanov et al., 2019), which have revolutionized cryo-EM, it is possible that the previous difficulties can now be overcome and it is thus important to evaluate $1 \mathrm{He}$ cooling for singleparticle cryo-EM.

We used mouse apoferritin expressed in Escherichia coli as a test specimen. In eukaryotic cells, ferritin catalyses the oxidation of iron(II) to iron(III), which is then stored in the oligomeric complex (Honarmand Ebrahimi et al., 2015). The octahedral ferritin oligomer is composed of 24 subunits assembled into a hollow sphere in the apo state (Honarmand Ebrahimi et al., 2015). Each subunit has a molecular weight of $\sim 21 \mathrm{kDa}$ and is composed mainly of five $\alpha$-helices, four of which form a bundle (Crichton \& Declercq, 2010).

Owing to its high symmetry, apoferritin has excellent qualities for single-particle cryo-EM, as it allows 24-fold averaging and avoids issues of preferred particle orientation. In these respects it is superior to other commonly used test specimens of similar size, such as $\beta$-galactosidase, which only features fourfold symmetry and tends to orient preferentially on cryo-grids. The cryo-EM structure of apoferritin has recently been refined to resolutions of $1.65 \AA$ (EMDB entry EMD-0144; Zivanov et al., 2018) and $1.62 \AA$ (EMDB entry EMD-9599), which are currently the highest resolutions reported for the technique, and apoferritin has been used as a test specimen in a number of methodological studies (Feng et al., 2017; Fan et al., 2017; Russo \& Passmore, 2014a,b; Zivanov et al., 2018; Marr et al., 2014). It is becoming a new standard and as such is the appropriate sample for this study.

\section{Materials and methods}

\subsection{Sample preparation and data collection}

Samples of mouse heavy-chain apoferritin (UniProt P09528) at $5.9 \mathrm{mg} \mathrm{ml}^{-1}$ in buffer (20 $\mathrm{m} M$ HEPES pH 7.5, $300 \mathrm{mM} \mathrm{NaCl}$ ) were diluted 1:4 in water and centrifuged to reduce aggregates in the supernatant. $3 \mu \mathrm{l}$ droplets were deposited onto glow-discharged 300 mesh gold grids with an R2/2 gold support (Quantifoil). A Vitrobot Mark IV was used for blotting at $10^{\circ} \mathrm{C}$ and $100 \%$ humidity with $10 \mathrm{~s}$ blot time and a blot force of -2 . Grids were plunge-frozen in liquid ethane. Images were recorded at $300 \mathrm{kV}$ acceleration voltage in a JEOL 3200 FSC microscope equipped with an in-column energy filter operated at $20 \mathrm{eV}$ and a Gatan $\mathrm{K} 2$ direct electron detector operated in counting mode. Eucentric height, focus and astigmatism were carefully adjusted and regularly controlled throughout data collection. A magnification of $30000 \times$, which corresponds to a calibrated pixel size of $1.12 \AA$, was used to collect $8 \mathrm{~s}$ dose-fractionated movies with $0.2 \mathrm{~s}$ frames at an electron flux of $\sim 9 \mathrm{e}^{-}$per pixel per second. Two data sets were collected from the same grid, selecting holes with suitable ice quality for imaging. For the first data set both cooling tanks of the microscope were filled with liquid nitrogen, such that the temperature readout on the microscope showed $78 \mathrm{~K}$ for the inner tank and $85 \mathrm{~K}$ for the specimen stage. In the second data collection the inner cooling tank was filled with liquid helium, producing a readout of $9 \mathrm{~K}$ for the inner tank and $17 \mathrm{~K}$ for the specimen stage. Images of $1 \mathrm{~N}_{2}$-cooled samples were collected on three different days and images of $1 \mathrm{He}$-cooled samples on two different days, within sessions of 4-6 $\mathrm{h}$ each. The contamination rate in the JEOL column is estimated at $4-5 \AA$ per hour. In between collections, the grid was stored in the $\mathrm{IN}_{2}$-cooled microscope side arm in order to reduce the buildup of amorphous ice. A total of 271 movies were collected with $\mathrm{lN}_{2}$ cooling and 233 movies with $1 \mathrm{He}$ cooling. Similar experiments were performed with horse apoferritin (Sigma) and with $\mathrm{F}_{420}$-reducing hydrogenase (Frh) from Methanothermobacter marburgensis (Mills et al., 2013; Allegretti et al., 2014).

\subsection{Image processing and data analysis}

Movies were processed with RELION-3.0 beta (Zivanov et al., 2018) using MotionCor2 v.1.0.0 (Zheng et al., 2017) for initial motion correction and Gctf v.1.06 (Zhang, 2016) for the estimation of CTF parameters. The known magnification 
distortion of the electron microscope $(2.57 \%$ at a minor axis angle of $35.3^{\circ}$ ) was not corrected because the current version of Bayesian polishing and CTF refinement in RELION-3 does not allow it. Particles were picked automatically using references from an initial set of manually picked particles. In order to discard false positives, a selection was performed via $2 \mathrm{D}$ classification. A total of 184777 particles for the data set

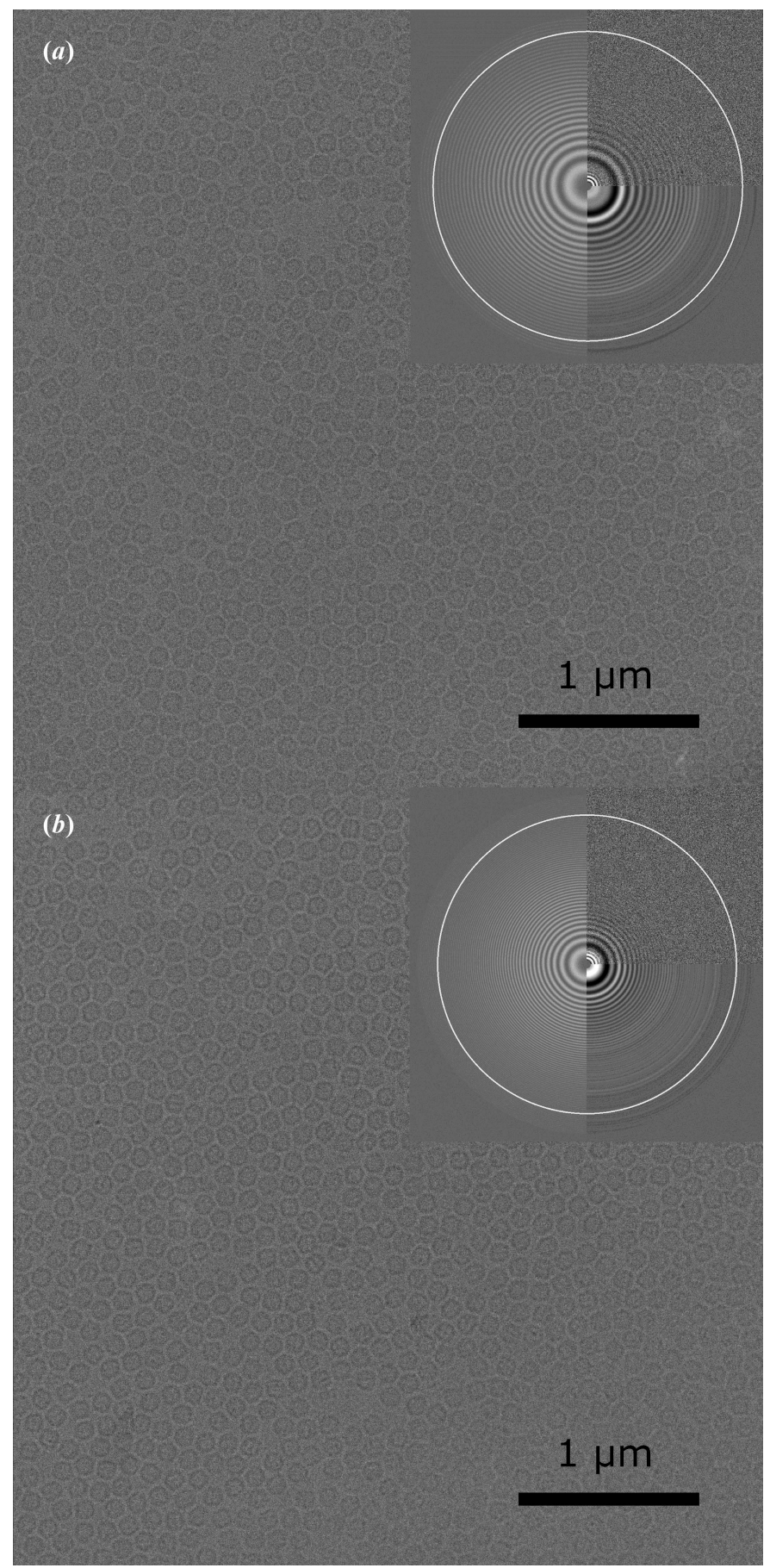

Figure 1

Exemplary micrographs with respective CTF estimations. (a) A micrograph acquired with liquid-nitrogen cooling, with an estimated defocus of $1.05 \mu \mathrm{m}$. The CTF goes out to $2.5 \AA$ (white ring). (b) A micrograph acquired with liquid-helium cooling, with an estimated defocus of $1.99 \mu \mathrm{m}$. The CTF goes out to $2.6 \AA$ (white ring). collected with $1 \mathrm{~N}_{2}$ cooling and 190785 particles for the data set collected with $1 \mathrm{He}$ cooling were extracted using box sizes of 176 pixels. Three-dimensional maps were refined using a deposited apoferritin map (EMDB entry EMD-9599) filtered to $10 \AA$ as an initial model. After this, Bayesian polishing and CTF refinement were iteratively applied in order to improve motion correction and CTF parameter estimation on a perparticle basis. The final resolutions of the reconstructions were estimated using two separate half sets according to the goldstandard 0.143 Fourier shell correlation (FSC) cutoff (Rosenthal \& Henderson, 2003) and the FSC between the two reconstructions was calculated. In a separate refinement, all
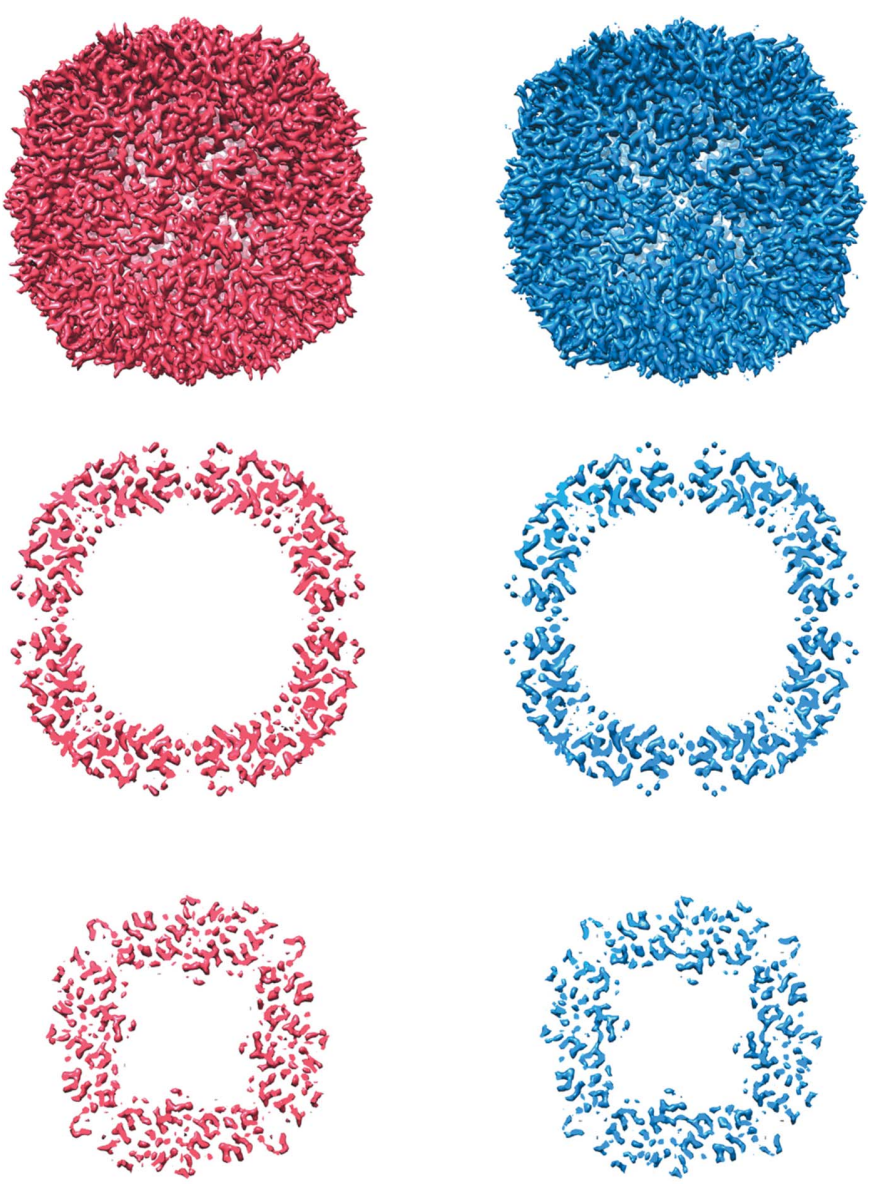

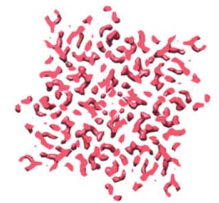

(a)

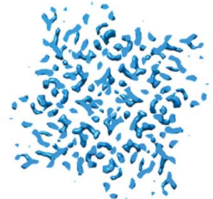

(b)
Figure 2

Refined cryo-EM maps and slices of mouse heavy-chain apoferritin from data acquired with liquid-nitrogen cooling $(a)$ at an estimated resolution of $2.74 \AA$ and liquid-helium cooling $(b)$ at an estimated resolution of $2.78 \AA$. 
particles in the $1 \mathrm{~N}_{2}$ data set with less than $0.85 \mu \mathrm{m}$ defocus were excluded. UCSF Chimera v.1.13.1 (Pettersen et al., 2004) was used for visualization of the Coulomb potential maps for control and comparison of the map qualities. Random subsets of $400,800,1600,3200,6400,12800,25600,51200$ or 102400 particles were individually refined for each data set and the inverse-squared resolutions obtained using these refinements were fitted to the natural logarithm of the number of particles using linear regression. Overall $B$ factors were then calculated by dividing 2 by the regression slopes (Zivanov et al., 2018). The standard errors of the regression slopes were calculated and propagated in order to estimate the errors in the $B$ factors. To estimate the motion of particles throughout the movies, coordinates from the Bayesian polishing feature in RELION-3 (Zivanov et al., 2019) were used to calculate Euclidean distances between particle positions in successive movie frames. These movements were added and averaged for all particles of each data set. Reconstructions containing only the information from single movie frames were produced by reextracting particles from single frames while keeping the particle-orientation information from previous refinements. Per-frame $B$ factors were calculated by the Bayesian polishing function within RELION-3.

All plots were produced with Matplotlib (Hunter, 2007) within Python. Figures showing Coulomb potential maps were produced in USCF Chimera v.1.13.1 (Pettersen et al., 2004).

\section{Results}

Two data sets of single-particle images of mouse heavy-chain apoferritin were collected at specimen temperatures of $85 \mathrm{~K}$ ( $\mathrm{IN}_{2}$ cooling) and $17 \mathrm{~K}$ (1He cooling) using a JEOL 3200 FSC electron microscope, in which the sample can be cooled with either $\mathrm{lN}_{2}$ or $\mathrm{lHe}$. Exemplary micrographs and CTF estimations are shown in Fig. 1. Image processing yielded reconstructions with final resolutions of $2.74 \AA$ for the data set

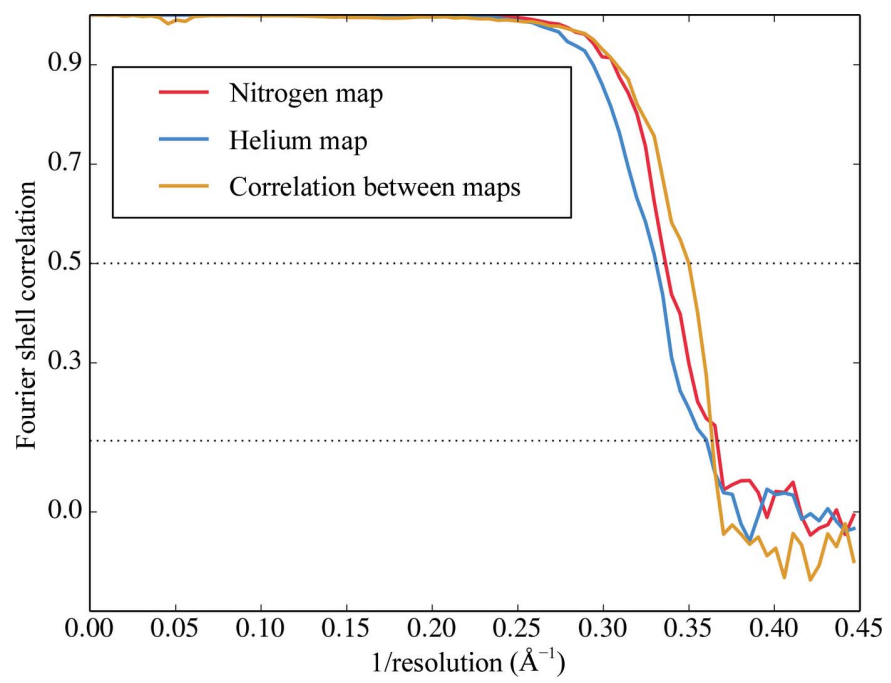

Figure 3

Fourier shell correlations (FSCs) of separately refined half maps from the data sets collected with $1 \mathrm{~N}_{2}$ cooling (FSC of 0.143 at $2.74 \AA$ ), lHe cooling (FSC of 0.143 at $2.78 \AA$ ) and between the two maps (FSC of 0.5 at $2.90 \AA$ ). collected at $85 \mathrm{~K}$ and $2.78 \AA$ for the data set collected at $17 \mathrm{~K}$. The reconstructed Coulomb potential maps are shown in Fig. 2 and respective Fourier shell correlations as well as the correlation between the final maps are shown in Fig. 3.

\subsection{Comparability of data sets}

The resolution achieved by cryo-EM depends on a number of factors, including sample quality, ice thickness, microscope alignment and imaging conditions. It is therefore important to ascertain that the two data sets are comparable. As a first measure, all data were collected from the same grid, ensuring consistency in the sample. Fig. 4 shows the estimated defocus values for all particles used in the reconstructions. The $\mathrm{lN}_{2}$ data set included images recorded closer to focus, which on the one hand might contribute more high-resolution information, but on the other hand have lower contrast, either of which

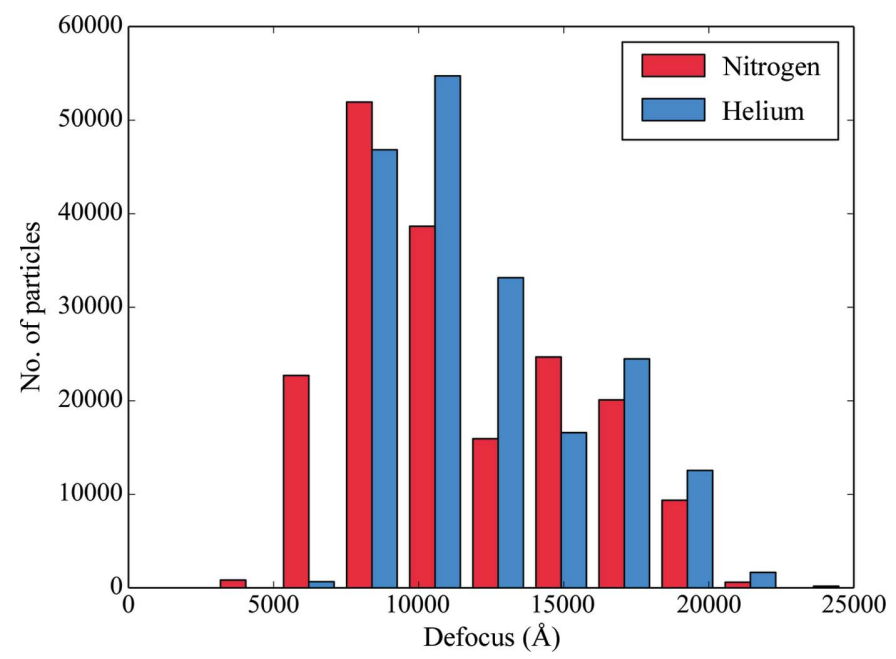

Figure 4

Histogram of defocus values of the particles used for reconstructions from data sets acquired with liquid-nitrogen or liquid-helium cooling.

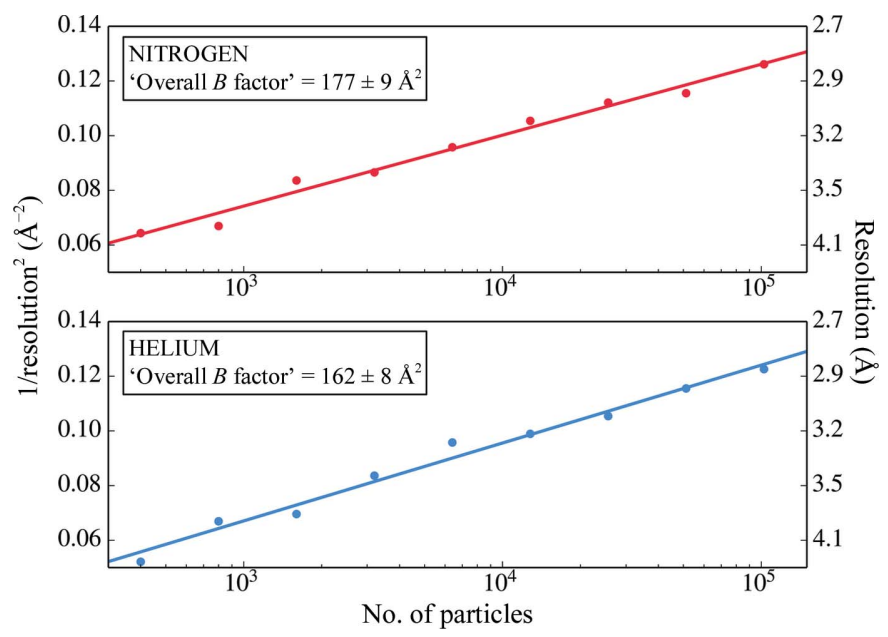

Figure 5

Squared inverse resolution achieved from random subsets of increasing numbers of particles (logarithmic scale) with linear fits for data sets acquired with $1 \mathrm{~N}_{2}$ and $\mathrm{lHe}$ cooling. From the slope, an 'overall $B$ factor' is determined. 
could bias the comparison. We ran a separate refinement that excluded all particles at less than $0.85 \mu \mathrm{m}$ defocus from the $\mathrm{lN}_{2}$ data set. The resolution remained unchanged at $2.74 \AA$, indicating that the defocus range that we used did not affect the map quality. In Fig. 5 the squared inverse resolution of reconstructions achieved from random subsets of particles is plotted against the subset size on a logarithmic scale. Theoretical considerations suggest this to be a linear relationship from which an 'overall $B$ factor' can be calculated for any given data set (Rosenthal \& Henderson, 2003). This $B$ factor relates the achieved resolution to the number of particles used in the reconstruction. By performing linear regression, overall $B$ factors of $177 \pm 9$ and $162 \pm 8 \AA^{2}$ were calculated for the data sets acquired with $\mathrm{lN}_{2}$ cooling and $\mathrm{lHe}$ cooling, respectively, showing that the data sets are of comparable quality. Table 1 gives a comparative overview of the two data sets.

\subsection{Beam-induced motion and radiation damage}

Fig. 6 shows that the beam-induced motion of particles in the first five or six image frames is substantially higher for 1 He-cooled samples. These frames are potentially the most precious for structure determination because they have suffered least from radiation damage. The Coulomb potential maps were compared with special attention to the carboxylate side chains of glutamates and aspartates (Fig. 7), which are known to suffer first from radiation damage (Vonck \& Mills, 2017). The map densities of these side chains are no better in the map derived from $1 \mathrm{He}$-cooled samples, which is not surprising as this type of radiation damage already occurs at doses equivalent to less than $1 \mathrm{e}^{-} \AA^{-2}$ (Henderson, 1990). As expected, other less radiation-sensitive side chains did not show significant differences in density either (not shown).

For further analysis of radiation damage, the resolutions of single-frame reconstructions and the relative per-frame $B$

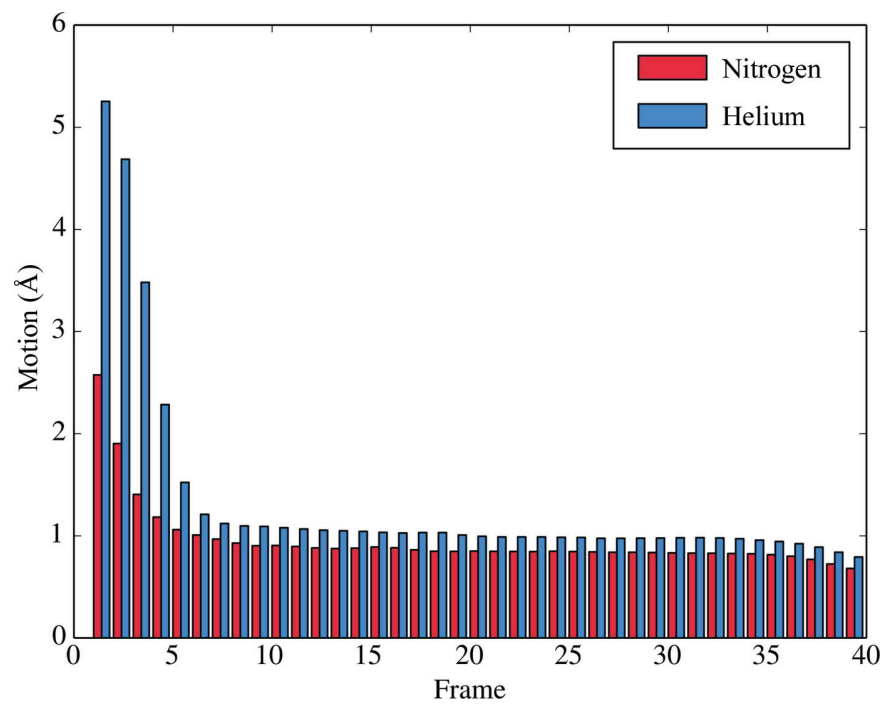

Figure 6

Average per-frame motion of particles imaged with $\mathrm{IN}_{2}$ or $\mathrm{lHe}$ cooling as determined by the Bayesian polishing algorithm within RELION-3. With lHe cooling, the motion in the first few frames is higher by a factor of more than 2 .

Table 1

Data collection and image processing.

\begin{tabular}{lll}
\hline & $\begin{array}{l}\text { Liquid-nitrogen } \\
\text { cooling }\end{array}$ & $\begin{array}{l}\text { Liquid-helium } \\
\text { cooling }\end{array}$ \\
\hline $\begin{array}{l}\text { Data collection } \\
\text { Microscope }\end{array}$ & JEOL 3200 FSC JEOL 3200 FSC \\
Voltage $(\mathrm{kV})$ & 300 & 300 \\
Temperature at specimen stage (K) & 85 & 17 \\
Camera & Gatan K2 & Gatan K2 \\
Calibrated pixel size $(\AA)$ & 1.12 & 1.12 \\
Electron flux $\left(\mathrm{e}^{-}\right.$per pixel per second) & 9 & 9 \\
Total exposure time $(\mathrm{s})$ & 8 & 8 \\
No. of frames per image & 40 & 40 \\
Dose per frame $\left(\mathrm{e}^{-} \AA^{-2}\right)$ & 1.43 & 1.43 \\
Defocus range $(\mu \mathrm{m})$ & $0.30-2.11$ & $0.66-2.47$ \\
No. of collected movies & 271 & 233 \\
Image processing & & \\
Motion-correction software & MotionCor 2 & MotionCor2 \\
CTF estimation software & Gctf & Gctf \\
Particle-selection software & RELION-3 & RELION-3 \\
No. of particle images & 148777 & 190785 \\
Final resolution $(\AA)$ & 2.74 & 2.78 \\
Applied sharpening $B$ factor $\left(\AA^{2}\right)$ & -126 & -128 \\
Estimated 'overall $B$ factor' $\left(\AA^{2}\right)$ & $177 \pm 9$ & $162 \pm 8$ \\
\hline
\end{tabular}

factors are shown in Fig. 8. These $B$ factors are estimated from the Fourier ring correlation between particle images and references as a part of the damage-weighting procedure in RELION-3 (Zivanov et al., 2019). They should not be confused with the 'overall $B$ factors' shown in Fig. 5 or the sharpening $B$ factors in Table 1 . As expected, later frames had more negative, i.e. worse, per-frame $B$ factors and lower resolution as a result of the cumulative effect of radiation damage. Surprisingly, $1 \mathrm{He}$ cooling proved to be worse both in terms of beam-induced movement (Fig. 6) and in terms of the resolution and decay of $B$ factors beyond frame $\sim 20$ (Fig. 8). An exception was the very first frame, which for $1 \mathrm{He}$ indicated a slightly better relative $B$ factor for unexplained reasons.

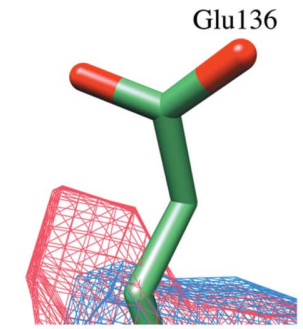
Asp146

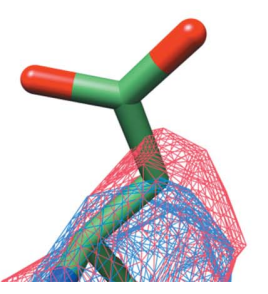

Figure 7

Selected carboxylate side chains showing signs of radiation damage. The reconstructed map from data acquired with $1 \mathrm{He}$ cooling (blue mesh) does not show better fits for the side chains of the fitted atomic model (PDB entry 3f32; Vedula et al., 2009) than the map from data acquired with $\mathrm{IN}_{2}$ cooling (red mesh). 

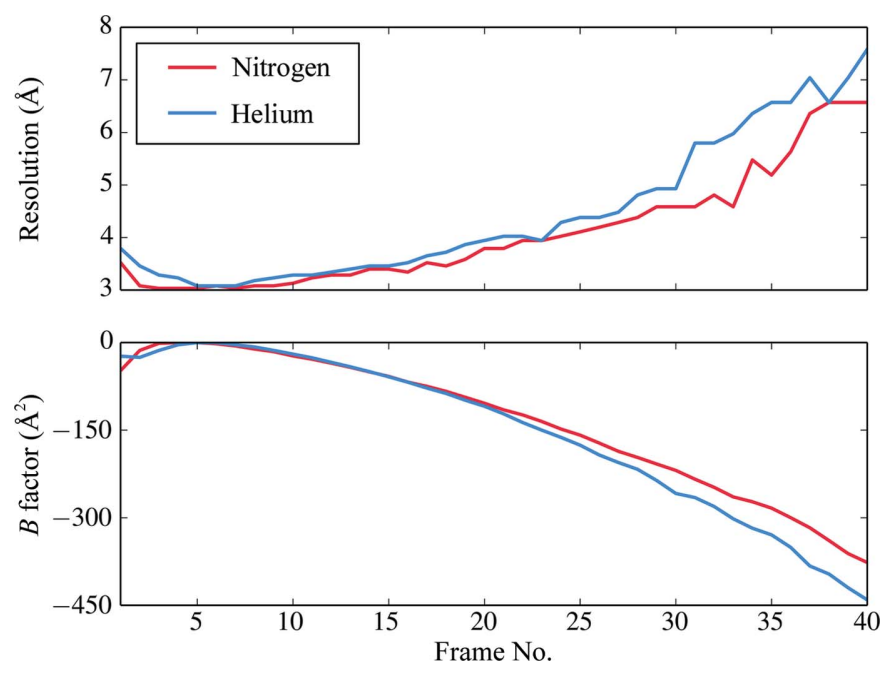

Figure 8

Resolutions achieved by reconstructions using only single frames of the recorded movies, keeping the optimized angles and positions from refinements using all frames (top), and per-frame $B$ factors as calculated with the Bayesian polishing function within RELION-3 (bottom) for data sets acquired with $\mathrm{lN}_{2}$ or $\mathrm{lHe}$ cooling.

\section{Discussion}

The aim of this study has been to examine whether lHe cooling offers any advantage for single-particle cryo-EM. Two data sets from the same grid of recombinant mouse heavy-chain apoferritin were collected at specimen temperatures of 85 or $17 \mathrm{~K}$, processed and compared.

Fig. 6 indicates two distinct phases of beam-induced movement. The first phase encompasses the first few frames for both $1 \mathrm{He}$ and $\mathrm{IN}_{2}$ cooling. For each frame the sample is exposed to a dose of $1.43 \mathrm{e}^{-} \AA^{-2}$ (Table 1). This low dose causes a movement of more than $5 \AA$ in the first frame for the lHe-cooled sample. The next frames are affected progressively less, but overall beam-induced movement in this initial phase is worse by a factor of two or more for $1 \mathrm{He}$ compared with $1 \mathrm{~N}_{2}$ cooling. Possible causes include specimen charging (Glaeser, 2016; Russo \& Henderson, 2018) and pre-existing mechanical stress frozen into the sample that is released upon radiation (Glaeser, 2016; Vinothkumar \& Henderson, 2016). Both effects are likely to be stronger with $1 \mathrm{He}$ cooling. Charging is expected to increase owing to the lower conductivity of water and carbon at very low temperatures. Another possible cause of beam-induced movement in this early phase is the postulated collapse of vitreous water into a higher-density phase at lHe temperature that has been reported at a dose below $3 \mathrm{e}^{-} \AA^{-2}$ (Wright et al., 2006). The rearrangement of surrounding water molecules during this density change would result in a net movement of the protein particles.

The second phase of beam-induced movement encompasses frames 7 to (in our experiments) 40. Even though in this second phase the average movement per frame (i.e. per $1.43 \mathrm{e}^{-} \AA^{-2}$ ) with $1 \mathrm{He}$ and $\mathrm{IN}_{2}$ is similar (Fig. 6), the per-frame $B$ factor and resolution (Fig. 8) are both considerably worse for $1 \mathrm{He}$ beyond frame 15 , at a cumulative dose of above $20 \mathrm{e}^{-} \AA^{-2}$. A possible explanation might be that radiolytic fragments of the protein and surrounding water, in particular molecular $\mathrm{H}_{2}$ (melting point $14 \mathrm{~K}$, boiling point $20.3 \mathrm{~K}$ ), are trapped in $1 \mathrm{He}$-cooled samples but can diffuse into the column vacuum at $1 \mathrm{~N}_{2}$ temperature (Laufer et al., 1987; Sandford \& Allamandola, 1993; Flournov et al., 1962). All other radiolytic products, such as oxygen, methane and ethane, are solids at lHe temperature (Vinothkumar \& Henderson, 2016). Radiolytic hydrogen may form nascent gas bubbles that expand with increasing dose, causing local movement and blurring the high-resolution signal. The nascent bubbles might correspond to an early stage of the larger bubbles observed by electron tomography of lHe-cooled samples (Iancu et al., 2006).

The loss of high-resolution information is thus more severe for 1 He cooling both in the initial phase of electron irradiation and at higher cumulative doses in the subsequent phase. We confirmed these findings in similar experiments with two other test specimens, Frh (Allegretti et al., 2014; Mills et al., 2013) and horse spleen apoferritin, which likewise indicated more beam-induced motion during the initial phase and substantially worse per-frame $B$ factors in the subsequent phase for $1 \mathrm{He}$ cooling, as well as a better $B$ factor for the very first frame (data not shown).

The best high-resolution information from dose-fractionated movies is expected to be found in the first few frames, where radiation damage is least severe. At present, the highresolution signal contained in these early frames is attenuated by specimen movement, and our analysis indicates that this effect is particularly severe in lHe-cooled samples. The expected benefit of $1 \mathrm{He}$ cooling in terms of cryoprotection, reduced radiation damage and improved signal-to-noise ratio was not observed in this study. We conclude that at present cooling with $\mathrm{lHe}$ is not beneficial for single-particle cryo-EM. Once the problems associated with beam-induced specimen movement have been resolved, the potential benefit of $1 \mathrm{He}$ cooling for high-resolution cryo-EM should be re-examined.

\section{Acknowledgements}

We thank Haruaki Yanagisawa from Tokyo University and Yongchan Lee at the Department of Structural Biology, MPI of Biophysics for providing mouse apoferritin plasmids, David Wöhlert for apoferritin expression and purification, Susann Kaltwasser for help in optimizing freezing conditions, JanPhilip Wieferig for providing scripts for analysis and Richard Henderson for discussions and for comments on the manuscript.

\section{Funding information}

The following funding is acknowledged: Max-Planck-Gesellschaft (award to Werner Kühlbrandt).

\section{References}

Allegretti, M., Mills, D. J., McMullan, G., Kühlbrandt, W. \& Vonck, J. (2014). Elife, 3, e01963.

Bai, X.-C., McMullan, G. \& Scheres, S. H. W. (2015). Trends Biochem. Sci. 40, 49-57. 
Bammes, B. E., Jakana, J., Schmid, M. F. \& Chiu, W. (2010). J. Struct. Biol. 169, 331-341.

Cheng, Y. (2015). Cell, 161, 450-457.

Comolli, L. R. \& Downing, K. H. (2005). J. Struct. Biol. 152, 149-156.

Crichton, R. R. \& Declercq, J.-P. (2010). Biochim. Biophys. Acta, 1800, 706-718.

Danev, R. \& Nagayama, K. (2008). J. Struct. Biol. 161, 211-218.

Fan, X., Zhao, L., Liu, C., Zhang, J.-C., Fan, K., Yan, X., Peng, H.-L., Lei, J. \& Wang, H.-W. (2017). Structure, 25, 1623-1630.

Feng, X., Fu, Z., Kaledhonkar, S., Jia, Y., Shah, B., Jin, A., Liu, Z., Sun, M., Chen, B., Grassucci, R. A., Ren, Y., Jiang, H., Frank, J. \& Lin, Q. (2017). Structure, 25, 663-670.

Flournov, J. M., Baum, L. H. \& Siegel, S. (1962). J. Chem. Phys. 36, 2229-2230.

Fujiyoshi, Y. (1998). Adv. Biophys. 35, 25-80.

Fujiyoshi, Y., Mizusaki, T., Morikawa, K., Yamagishi, H., Aoki, Y., Kihara, H. \& Harada, Y. (1991). Ultramicroscopy, 38, 241-251.

Glaeser, R. M. (2016). Methods Enzymol. 579, 19-50.

Gonen, T., Cheng, Y., Sliz, P., Hiroaki, Y., Fujiyoshi, Y., Harrison, S. C. \& Walz, T. (2005). Nature (London), 438, 633-638.

Hayward, S. B. \& Glaeser, R. M. (1979). Ultramicroscopy, 4, 201-210.

Henderson, R. (1990). Proc. R. Soc. London Ser. B, 241, 6-8.

Henderson, R., Baldwin, J. M., Ceska, T. A., Zemlin, F., Beckmann, E. \& Downing, K. H. (1990). J. Mol. Biol. 213, 899-929.

Hiroaki, Y., Tani, K., Kamegawa, A., Gyobu, N., Nishikawa, K., Suzuki, H., Walz, T., Sasaki, S., Mitsuoka, K., Kimura, K., Mizoguchi, A. \& Fujiyoshi, Y. (2006). J. Mol. Biol. 355, 628-639.

Holm, P. J., Bhakat, P., Jegerschöld, C., Gyobu, N., Mitsuoka, K., Fujiyoshi, Y., Morgenstern, R. \& Hebert, H. (2006). J. Mol. Biol. 360, 934-945.

Honarmand Ebrahimi, K., Hagedoorn, P.-L. \& Hagen, W. R. (2015). Chem. Rev. 115, 295-326.

Hunter, J. D. (2007). Comput. Sci. Eng. 9, 90-95.

Iancu, C. V., Wright, E. R., Heymann, J. B. \& Jensen, G. J. (2006). J. Struct. Biol. 153, 231-240.

Jeng, T.-W. \& Chiu, W. (1984). J. Microsc. 136, 35-44.

Jiang, W., Baker, M. L., Jakana, J., Weigele, P. R., King, J. \& Chiu, W. (2008). Nature (London), 451, 1130-1134.

Kimura, Y., Vassylyev, D. G., Miyazawa, A., Kidera, A., Matsushima, M., Mitsuoka, K., Murata, K., Hirai, T. \& Fujiyoshi, Y. (1997). Nature (London), 389, 206-211.

Kühlbrandt, W. (2014a). Elife, 3, e03678.

Kühlbrandt, W. (2014b). Science, 343, 1443-1444.

Kühlbrandt, W., Wang, D. N. \& Fujiyoshi, Y. (1994). Nature (London), 367, 614-621.

Laufer, D., Kochavi, E. \& Bar-Nun, A. (1987). Phys. Rev. B, 36, 9219 9227.
Ludtke, S. J., Baker, M. L., Chen, D.-H., Song, J.-L., Chuang, D. T. \& Chiu, W. (2008). Structure, 16, 441-448.

Marr, C. R., Benlekbir, S. \& Rubinstein, J. L. (2014). J. Struct. Biol. 185, 42-47.

Meents, A., Gutmann, S., Wagner, A. \& Schulze-Briese, C. (2010). Proc. Natl Acad. Sci. USA, 107, 1094-1099.

Mills, D. J., Vitt, S., Strauss, M., Shima, S. \& Vonck, J. (2013). Elife, 2, e00218.

Miyazawa, A., Fujiyoshi, Y. \& Unwin, N. (2003). Nature (London), 423, 949-955.

Murata, K., Mitsuoka, K., Hirai, T., Walz, T., Agre, P., Heymann, J. B., Engel, A. \& Fujiyoshi, Y. (2000). Nature (London), 407, 599-605.

Pettersen, E. F., Goddard, T. D., Huang, C. C., Couch, G. S., Greenblatt, D. M., Meng, E. C. \& Ferrin, T. E. (2004). J. Comput. Chem. 25, 1605-1612.

Quentin, D. \& Raunser, S. (2018). J. Mol. Med. 96, 483-493.

Rosenthal, P. B. \& Henderson, R. (2003). J. Mol. Biol. 333, 721745.

Russo, C. J. \& Henderson, R. (2018). Ultramicroscopy, 187, 43-49.

Russo, C. J. \& Passmore, L. A. (2014a). Nat. Methods, 11, 649-652.

Russo, C. J. \& Passmore, L. A. (2014b). Science, 346, 1377-1380.

Sandford, S. A. \& Allamandola, L. J. (1993). Astrophys. J. 409, L65.

Sato, C., Ueno, Y., Asai, K., Takahashi, K., Sato, M., Engel, A. \& Fujiyoshi, Y. (2001). Nature (London), 409, 1047-1051.

Scapin, G., Potter, C. S. \& Carragher, B. (2018). Cell. Chem. Biol. 25, 1318-1325.

Stark, H., Zemlin, F. \& Boettcher, C. (1996). Ultramicroscopy, 63, 7579.

Tani, K., Mitsuma, T., Hiroaki, Y., Kamegawa, A., Nishikawa, K., Tanimura, Y. \& Fujiyoshi, Y. (2009). J. Mol. Biol. 389, 694-706.

Unwin, N. (2005). J. Mol. Biol. 346, 967-989.

Unwin, P. N. T. \& Henderson, R. (1975). J. Mol. Biol. 94, 425-440.

Vedula, L. S., Brannigan, G., Economou, N. J., Xi, J., Hall, M. A., Liu, R., Rossi, M. J., Dailey, W. P., Grasty, K. C., Klein, M. L., Eckenhoff, R. G. \& Loll, P. J. (2009). J. Biol. Chem. 284, 24176-24184.

Vinothkumar, K. R. \& Henderson, R. (2016). Q. Rev. Biophys. 49, e13.

Vonck, J. \& Mills, D. J. (2017). Curr. Opin. Struct. Biol. 46, 48-54.

Wright, E. R., Iancu, C. V., Tivol, W. F. \& Jensen, G. J. (2006). J. Struct. Biol. 153, 241-252.

Zhang, K. (2016). J. Struct. Biol. 193, 1-12.

Zheng, S. Q., Palovcak, E., Armache, J.-P., Verba, K. A., Cheng, Y. \& Agard, D. A. (2017). Nat. Methods, 14, 331-332.

Zhou, Z. H. (2008). Curr. Opin. Struct. Biol. 18, 218-228.

Zivanov, J., Nakane, T., Forsberg, B. O., Kimanius, D., Hagen, W. J. H., Lindahl, E. \& Scheres, S. H. W. (2018). Elife, 7, e42166.

Zivanov, J., Nakane, T. \& Scheres, S. H. W. (2019). IUCrJ, 6, 5-17. 